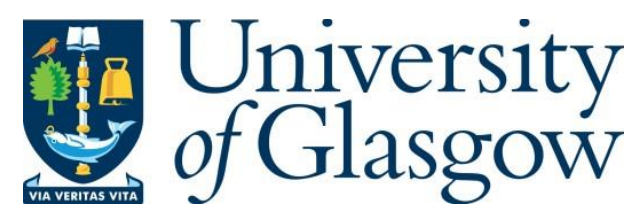

Rubio-Gracia, J. et al. (2018) Prevalence, predictors and clinical outcome of residual congestion in acute decompensated heart failure. International Journal of Cardiology, 258, pp. 185-191.

There may be differences between this version and the published version. You are advised to consult the publisher's version if you wish to cite from it.

http://eprints.gla.ac.uk/163234/

Deposited on: 31 May 2018

Enlighten - Research publications by members of the University of Glasgow http://eprints.gla.ac.uk 


\section{Prevalence, predictors and clinical outcome of residual congestion in Acute Decompensated Heart Failure}

Jorge Rubio-Gracia ${ }^{1,2,3}$, Biniyam G. Demissei ${ }^{3}$, Jozine M. ter Maaten ${ }^{3}$,John G. Cleland ${ }^{4}$, Christopher M. O'Connor ${ }^{5}$, Marco Metra ${ }^{6}$, Piotr Ponikowski ${ }^{7}$, John R. Teerlink ${ }^{8}$, Gad Cotter ${ }^{9}$, Beth A. Davison ${ }^{9}$, Michael M. Givertz ${ }^{10}$, Daniel M. Bloomfield ${ }^{11}$, Howard Dittrich ${ }^{12}$, Kevin Damman $^{3}$, Juan I. Pérez-Calvo ${ }^{1,2}$, Adriaan A. Voors ${ }^{3}$.

1.Servicio de Medicina Interna. Hospital Clínico Universitario “Lozano Blesa”. Zaragoza. Spain.

2.Instituto de Investigación Sanitaria de Aragón. Zaragoza. Spain.

3. University of Groningen. Department of Cardiology. University Medical Center Groningen. Groningen. The Netherlands.

4.Imperial College, London, UK

5.Inova Heart and Vascular Institute, Falls Church, VA, USA

6.University of Brescia, Brescia, Italy

7.Medical University, Clinical Military Hospital, Wroclaw, Poland

8.University of California at San Francisco and San Francisco Veterans Affairs Medical Center, San Francisco, CA, USA

9.Momentum Research Inc., Durham, NC, USA

10.Brigham and Women's Hospital, Boston, MA, USA

11.Merck \& Co., Inc. Kenilworth, NJ USA

12.Abboud Cardiovascular Research Center, University of lowa Carver College of Medicine, lowa, USA

Word count: 3041

Corresponding Author:

Adriaan A. Voors M.D. PhD.

Department of Cardiology, University Medical Center Groningen.

Groningen. The Netherlands.

Hanzeplein 1, 9713 GZ Groningen

Tel: +31 503612355. Fax: +31 503614391. E-mail: a.a.voors@umcg.nl 
Background: Congestion is the main reason for hospital admission for acute decompensated heart failure (ADHF). A better understanding of the clinical course of congestion and factors associated with decongestion are therefore important. We studied the clinical course, predictors and prognostic value of congestion in a cohort of patients admitted for ADHF by including different indirect markers of congestion (residual clinical congestion, Brain natriuretic peptides (BNP) trajectories, hemoconcentration or diuretic response).

\section{Methods and results: We studied the prognostic value of residual clinical congestion} using an established composite congestion score (CCS) in 1572 ADHF patients. At baseline, 1528 (97.2\%) patients were significantly congested (CCS $\geq 3$ ), after 7 days of hospitalization or discharge (whichever came first), 451 (28.7\%) patients were still significantly congested (CCS $\geq 3), 751(47.8 \%)$ patients were mildly congested (CCS $=1$ or 2) and $370(23.5 \%)$ patients had no signs of residual congestion (CCS=0). The presence of significant residual congestion at day 7 or discharge was independently associated with increased risk of re-admissions for heart failure by day $60(\mathrm{HR}[95 \% \mathrm{Cl}]=1.88$ [1.39-2.55]) and all-cause mortality by day $180(\mathrm{HR}[95 \% \mathrm{Cl}]=1.54$ [1.16-2.04]). Diuretic response provided added prognostic value on top of residual congestion and baseline predictors for both outcomes, yet gain in prognostic performance was modest.

Conclusion: Most patients with acute decompensated heart failure still have residual congestion 7 days after hospitalization. This factor was associated with higher rates of re-hospitalization and death. Decongestion surrogates, such as diuretic response, added to residual congestion, are still significant predictors of outcomes, but they do not provide meaningful additive prognostic information. 
KEY WORDS: Heart Failure; Congestion; Diuretic response. 


\section{Introduction}

Most patients needing hospital admission for decompensated heart failure present with signs and symptoms of congestion [1]. Relief of signs and symptoms of congestion (i.e. decongestion) is one of the main goals of in-hospital treatment in these patients $[2,4,5]$.

However, adequate decongestion is often not achieved during hospital admission. In a recent post-hoc analysis of DOSE-AHF and CARESS-HF $[6,7]$, only half of the patients were free from signs of congestion at discharge, and these patients had lower rates of death and re-hospitalization at day 60 . Similarly, using a composite congestion score (Supplementary table 1) that comprised orthopnea, jugular venous distension (JVD) and peripheral edema, Ambrosy et al [7] showed that a significant proportion of patients still had residual congestion by day 7 or discharge, and these patients had increased risks of readmission and mortality.

Improved clinical assessment of residual congestion is therefore paramount, and a better understanding of the clinical course of congestion and factors associated with decongestion could play an important role towards the implementation of targeted strategies that can reduce residual congestion and, potentially, improve outcomes [8]. Nonetheless, assessment of decongestion based strictly on clinical findings may be non-sensitive. It has been shown that the change in BNP concentrations [9] and hematocrit during hospitalization [10], as surrogates markers of congestion, add significant prognostic information related with residual congestion. In addition, the metrics of diuretic response seems to be crucial in achieving a safe decongestion $[8,11-13,28]$. The combination of objective measures of decongestion on top of clinical 
assessment may help to detect lesser degrees of congestion and lead to a more accurate and safe treatment and follow-up.

We therefore aimed to: (i) characterize the clinical course of congestion (ii) evaluate predictors of residual congestion by day 7 (iii) assess the prognostic value of residual congestion by day 7 and (iiii) evaluate the prognostic value of decongestion markers along with clinical findings of residual congestion in patients hospitalized for decompensated heart failure.

\section{Methods}

\section{Study population and procedures}

Data from PROTECT (Placebo-controlled Randomized Study of the Selective A1 Adenosine Receptor Antagonist Rolofylline for Patients Hospitalized with Acute Decompensated Heart Failure and Volume Overload to assess Treatment Effect on congestion and Renal function) were utilized in this study $[14,15]$. The PROTECT trial was designed to study rolofylline, an adenosine A1-receptor antagonist as a new treatment for ADHF capable of improving renal function and relieving dyspnea. Main inclusion criteria were persistent dyspnea at rest, impaired renal function, high titers of natriuretic peptides, ongoing intravenous loop diuretic therapy and enrollment within 24 hours after admission [11,14]. Other inclusion and exclusion criteria have been previously described [14]. The main findings were neutral with respect to the primary outcome [11].

Clinical assessment of symptoms and signs, including orthopnea, rales, edemas, JVD, dyspnea and body weight was performed daily by clinicians until day 7 or discharge (if 
earlier), and day 14. Diuretic response was calculated as $\Delta$ body weight in the first 72 hours/40mg i.v. furosemide or equivalent [28]. Standard laboratory parameters were measured in a central laboratory (ICON laboratories, Farmingdale, New York).

\section{Composite congestion score}

A composite congestion score was calculated for individual patients at baseline and on days $2,3,4,7$ and 14 using a modified algorithm from the one described by Ambrosy et al [7]. The composite congestion score was calculated by summing the individual scores for orthopnea (0 to 3 ), peripheral edema (0 to 3 ) and JVD (0 to 2 ). In contrast to the previously published composite congestion score [7], the maximum possible score was 8 points in the current study as the coding of JVD was slightly different in the PROTECT trial (Supplementary table 2).

\section{Surrogate markers of decongestion}

We, thereafter, included several objective measurements of decongestion on top of the aforementioned clinical findings.

Changes in concentration of BNP was defined as (BNP day 7 or discharge-BNP day 1), To calculate percentage change in brain natriuretic peptide (BNP) till day 7 or day 14 from baseline, we used non-commercial plasma BNP measured using a single molecule counting technology with the Erenna ${ }^{\circledR}$ Immunosay System on a microtitre plate assay format from frozen plasma samples (Singulex Inc., Alameda, CA, USA). BNP at baseline was available in 1,585 patients. BNP at day 7 was available in 1,442 patients. 1,248 patients had complete BNP data available on both time points. 
Hemoconcentration was defined as the change in hemoglobin at discharge or day 7 and diuretic response was calculated as $\Delta$ body weight in the first 72 hours/40mg i.v. furosemide or equivalent [28]. Also, change in estimated plasma volume (delta ePVS) was evaluated. [29]

\section{Study outcomes}

Two time-to-event outcomes, heart failure re-hospitalization by day 60 and all-cause mortality by day 180 were assessed. Follow-up for these analyses started at day 7, as the follow-up started before the end of the index hospitalization, we did not report death during hospitalization as these were included in the all-cause mortality by day 180 endpoint. All re-hospitalizations after index hospitalization and all causes of death through day 60 had been adjudicated by an independent clinical events committee.

\section{Statistical analysis}

Baseline clinical characteristics and standard laboratory parameters were summarized and compared in three groups based on composite congestion score on day 7 ( $0=$ no congestion, $1-2=$ mild congestion and $3-8=$ significant congestion). Continuous variables were summarized as mean \pm SD or median (interquartile range) as appropriate. ANOVA (for normally distributed variables) or Kruskall-Wallis (for nonnormally distributed variables) tests were used for group comparisons. Categorical variables were compared among groups with the chi-square test. No imputations were performed for missing values. 
The clinical course of congestion within the first 14 days of the index hospitalization was graphically assessed by plotting the proportion of patients within each of the three groups over multiple time points; baseline and 2, 3, 4, 7 and 14.

\section{A multivariable explanatory logistic regression model was developed to identify} factors independently associated with the presence of significant residual congestion by day 7. Candidate predictors were first selected based on a p-value $<20 \%$, next utilizing an Akaike information criterion (AIC) based backward selection procedure. An internal bootstrap with 1000 replicates of the selected models was performed, testing stability of these models. List of candidate variables considered for this model are included in supplementary material. Before the implementation of the stepwise selection procedure, linearity of association between baseline parameters and residual congestion by day 7 was evaluated using fractional polynomials and appropriate transformations were performed as necessary.

Unadjusted associations between the presence of significant residual congestion by day 7 and clinical outcomes were assessed using univariable cause-specific Cox proportional hazards models. The assumption of proportional hazards was checked and satisfied. Adjusted associations were further evaluated with multivariable causespecific Cox proportional hazards models that included previously identified predictors for the 180-day all-cause mortality outcome [15]. These encompassed baseline variables including age, peripheral edema, past heart failure hospitalization, systolic blood pressure (SBP), serum creatinine, blood urea nitrogen (BUN), albumin, sodium. For the 60-day heart failure rehospitalization outcome, a baseline model encompassing history of diabetes mellitus, percutaneous intervention (PCI), COPD, 
coronary artery bypass graft (CABG), heart failure hospitalization within the past year, albumin, BUN, hematocrite, sodium, edema and JVD was developed after implementation of a AIC-based backward selection procedure on a Cox regression model that included candidate predictors associated with outcome at a significance level of $20 \%$. This procedure was performed in multiple bootstrap samples using $R$ package bootStepAIC.

Unadjusted and adjusted associations between the other decongestion markers (i.e. change in BNP from baseline to day 7, diuretic response and hemoconcentration) were assessed in univariable and multivariable cause-specific proportional hazards models. Adjusted associations were evaluated in multivariable models that include the previously defined baseline predictors of each outcome and residual clinical congestion at day 7. Added prognostic value was quantified with the gain in the Harrell's C-index.

Estimates are presented with $95 \%$ confidence intervals. P-value $<0.05$ was considered statistically significant. Statistical analyses were performed with SPSS version 22.0 (IBM Corp., Armonk, NY) and R: Language and Environment for Statistical Computing, version 3.0.2. (R Foundation for Statistical Computing, Vienna, Austria).

\section{$\underline{\text { Results }}$}

\section{Baseline characteristics}

Of the 2033 patients included in PROTECT, 1572 patients had complete available assessment of orthopnea, JVD and peripheral edema at day 7. Patients with missing values were comparable to patients with available measurements (See Supplementary 
tables $3 \& 4)$. The majority of patients were male (67.1\%), with a mean age of $70.1 \pm$ 11.5 years, and had a previous history of heart failure hospitalizations $(94.8 \%)$. hypertension (79.4\%), ischemic heart disease (69.7\%), hyperlipidemia (51.9\%), atrial/flutter fibrillation (54.6\%) and diabetes (45.4\%). (Table 1).

\section{Evolution of composite congestion score during 14 days of baseline}

\section{assessment}

Nearly all patients included in the study (97.2\%) had moderate to severe congestion at baseline as assessed by the composite clinical congestion score. At baseline, the median [IQR] composite congestion score was 5 [4-6]. A significant reduction in the composite congestion score was observed during the next 7 days after baseline assessment (Supplementary figure 1). The median [IQR] composite congestion score declined from 5 to 2 [1-3] by day 7. However, 29\% of patients still had significant residual congestion by day 7 or discharge while more than $75 \%$ had a composite congestion score $>0$. The composite congestion score further declined to a median [IQR] value of 1 [0-3] by day 14 although $25 \%$ of evaluated patients still had significant residual congestion (Supplementary figure 1).

\section{Factors associated with the presence of significant congestion by day 7}

Table 1 shows baseline characteristics of patients with no, moderate, significant clinical congestion at day 7. Patients with significant residual congestion by day 7 had higher BMI [29.5 [26.1-34.5); $p=<0.001]$, lower systolic and diastolic blood pressure [120 (109.0-138.0); $p=0.011]-[71(63.0-80.0) ; p=0.017]$ and showed a higher prevalence of hyperlipidemia (59.3\%; $p=<0.001$ ), diabetes (54.3\%; $p=<0.001$ ), ischemic coronary 
disease (24.4\%; $p=<0.001)$ and CABG (27.5\%; $p=<0.001)$. Also, patients with significant clinical congestion at day 7 had higher BNP levels at day 7 and showed significantly less hemoconcentration (supplementary table 7).

Higher baseline BUN levels, poorer diuretic response, lower ALT and total cholesterol, higher BMI and BNP and a history of pacemaker implantation were found to be independent predictors of the presence of significant residual clinical congestion by day 7 (Table 2) This model had an AUC of 0.80.

\section{Residual clinical congestion and outcomes}

Among the 1572 patients who had composite congestion score assessment by day 7 , $15.8 \%(\mathrm{~N}=249)$ died by day 180 and $13.7 \%(\mathrm{~N}=215)$ were re-hospitalized for heart failure at least once by day 60 . Using Fine-Gray analysis we did not find a significant effect of competing risk ( $P$-0.11, supplementary figure 2$)$.

As presented in the Kaplan-Meier plots in Figures $1 \& 2$, the rates of both all-cause mortality by day 180 and heart failure re-hospitalization by day 60 increased significantly with increasing severity of residual clinical congestion by day 7 . The risk of all-cause mortality by day 180 more than doubled in patients with significant congestion by day 7 compared to those with no or mild congestion, (Hazard Ratio (HR) $2.13,95 \% \mathrm{Cl}[1.66-2.73])$. The risk of heart failure re-hospitalization by day 60 was also significantly greater in patients with significant residual congestion by day 7, (HR 1.88, 95\% $\mathrm{Cl}$ [1.43-2.46]). The risk associated with significant residual congestion by day 7 remained significantly increased even after adjustment for the baseline risk prediction models (Supplementary table 5). 
Added prognostic value of other decongestion markers on top of residual clinical congestion

Diuretic response was independently predictive of both HF-rehospitalization by day 60 and all-cause mortality by day 180 on top of residual clinical congestion and the baseline models. However, the gain the Harrell's C-index was modest for both outcomes (Supplementary Table 6).

On the other hand, hemocentration and also delta estimated plasma volume was strongly associated with all-cause mortality by day 180, yet the gain in the Harrell's C-index was modest. (Supplementary Table 6)

\section{Discussion}

In the present study, we demonstrate that one third of patients were still significantly congested at day 7. Residual congestion was associated with increased risks of death and heart failure re-hospitalization.

Our findings support previous results reported from the DOSE, CARRESS-HF and EVEREST trials, showing that residual congestion at day 7 or discharge is present in most patients admitted for ADHF and that residual congestion was related to poor clinical outcome $[6,7,16,17]$. In addition, consistent with previous studies, we identified higher BMI, BUN and the presence of comorbidities (e.g. diabetes) as important predictors of residual congestion [6]. Besides validating the existing body of evidence on clinically assessed residual congestion in ADHF patients, the current study 
provides novel information. The clinical course of congestion was more extensively characterized during hospitalization as physician assessment of clinical congestion was performed daily through day 4 in most patients included in the PROTECT trial. Interestingly, a significant decline in the composite clinical congestion score was already observed by day 4 as more than half of patients with significant congestion during baseline assessment regressed to no/mild congestion by day 4 . However, regardless of the rapid improvement of congestion by day 4 , no differences were found in length of hospital stay when patients were classified by their day 7 congestion score. This indicates that the decision to discharge patients is less likely to be driven by residual clinical congestion status.

Finally, residual congestion was also linked to heart failure treatment. Patients with some grade of residual congestion at day 7 or discharge, were being treated in a lower proportion with ACEi/ARBs $(p=0.017)$. Probably some factors as hypotension or a poorer renal function can explain this situation, those patients being "cold and wet" at physical examination have worse prognosis, indicating that they have and advanced heart failure situation and are severely congested.

Predictors of residual congestion

The strongest independent predictors of residual congestion were higher BUN on admission, a more severe clinical congestion at baseline and a poorer diuretic response.

Diuretic response reflects the effectiveness of loop diuretics to force diuresis and natriuresis $[12,18,19]$. A poorer diuretic response has been associated with more 
advanced heart failure, residual congestion and renal impairment, being predictive for mortality and heart failure re-hospitalization [11,13,18-21].

Blood urea nitrogen (BUN) is directly linked with diuretic response. Firstly, higher BUN is a marker of renal dysfunction, and renal dysfunction is a predictor of a poor diuretic response. Secondly, BUN is a marker of neurohormonal activation, which increases renal water and sodium retention to recover cardiac output when myocardial injury is developed [23-25]. The chronic neurohormonal activation leads to interstitial volume expansion and increase of central venous pressure, which in essence, is the definition of congestion [24]. Thirdly, BUN might directly have a negative effect in tubular response, since it is bound to the organic anion transporter [12]. Diuretic response and BUN therefore overlap, but were also independent predictors of residual congestion at day 7 or discharge.

In the multivariable analysis, body mass index (BMI), was also selected as an independent predictor for residual congestion, but this result must be interpreted with caution. Higher weight might simply reflect more congestion [24-26].

Lower total cholesterol levels were also independently associated with residual clinical congestion, possibly since more congested patients were more often patients with ischemic heart disease, and they might have a tighter control of total cholesterol levels. Lower total cholesterol levels might also be associated cachexia and of disease severity. 
We have found a strong association between clinical residual congestion and clinical outcome. The increased risk of hospital readmission in patients with more residual congestion is of particular interest, since it is more difficult to predict hospital readmission than death, and this particular association suggests that congestion should be studied as a marker of intensified decongestion [27], potentially leading to a reduction of early post-discharge hospital readmission.

Different models based on congestion signs and symptoms have been applied before with good results [6-7]. However, when using these models to assess congestion during an ADHF admission to predict HF-rehospitalizations, our results are in line with those found in DOSE-HF and CARESS-HF trials [7], confirming that there is a considerable group of patients who still have a high grade of residual congestion at discharge, probably because we adjust HF-treatment using an imprecise tool such as physical examination, which depends on the skills and experience of the physician.

For that purpose, several objective congestion markers as hemoconcentration, reduction in BNP [9], and diuretic response [12,18-19] have been included to assess whether they improved prognostic capacity of clinical congestion findings alone. Although all of them were significantly associated with residual congestion, and thus with prognosis, they actually did not provide relevant improvement in prognostic performance on top of baseline predictors and residual congestion for both heart failure re-admission and mortality outcomes. These results underline the importance of a thorough physical examination before discharge for an episode of ADHF. 
Our study suggests that strategies to improve decongestion and to discharge patients with less signs and symptoms of congestion might be a therapeutic target. However, prospective interventional studies are needed to establish whether decongestion might be a therapeutic target in patient with a hospital admission for heart failure.

\section{Limitations}

This study is a post-hoc analysis of a randomized clinical trial (PROTECT), with all limitations as such. For calculating the composite congestion score an important group of patients were lost during the analysis $(n=461)$. Besides, data on signs and symptoms of congestion were not collected per protocol at discharge, which could have added additional value to the analysis.

In addition, the analysis of added prognostic value of decongestion surrogates on top of residual clinical congestion was performed in different subsets of patients for each decongestion surrogate depending on the availability of data for each of the surrogates; performance of the baseline model + residual clinical congestion was slightly different across the subsets which might explain some of the discrepancies between the C-index and gain in C-index across the estimates for the different decongestion parameters (Suplemmentary table 5).

\section{Conclusions}

Residual congestion at day 7 or discharge was frequently found in patients with a hospital admission for ADHF. The strongest predictors of the presence of significant residual congestion by day 7 were more congestion at baseline, a poorer diuretic 
response and increased BUN. Patients who had significant congestion by day 7 had worse outcomes in terms of mortality and re-hospitalization for ADHF.

Surrogate markers of congestion as diuretic response had limited additive value to better predict HF-outcomes.

\section{Acknowledgements}

Singulex kindly provided assays and performed biomarker measurements.

\section{Funding}

The PROTECT trial was supported by NovaCardia, a subsidiary of Merck.

\section{Conflict of interest}

Beth Davison and Gad Cotter are employees of Momentum Research Inc., which has provided consulting services to NovaCardia, Merck, Corthera, Novartis, Singulex, ChanRx, Laguna Pharmaceuticals, Sorbent Therapeutics, Celyad SA, Trevena, Amgen, and Anexon. Marco Metra has received honoraria and reimbursements from NovaCardia, sponsors of the study, and from Merck, which purchased the rights to rolofylline after completion of the PROTECT pilot study. Daniel Bloomfield is an employee of Merck \& Co. John Cleland reports grants and personal fees from MSD, while conducting the study, grants and personal fees from Amgen, grants and personal fees from Novartis, personal fees from Stealth Biopharmaceuticals, personal fees from Servier, grants and personal fees from Bayer, and personal fees from Sorin, outside the submitted work. Howard Dittrich was an employee of NovaCardia and a consultant to Merck. Michael Givertz. has received institutional research support and served on a 
scientific Advisory Board for Merck. Piotr Ponikowski. has received honoraria from Merck, consulting fees from Vifor Pharma and Amgen, Inc., honoraria from Vifor Pharma, and travel/accommodation expenses covered by Vifor Pharma and Amgen, Inc. John Teerlink has received research funds and consulting fees from Merck, the makers of rolofylline for conducting this study and has also received research funds and/or consulting fees from Amgen, Cytokinetics, Novartis, Relypsa, Trevena, and ZS Pharma for research in related areas. Adriaan Voors has received speaker/consultancy/research fees from AstraZeneca, Bayer, BMS, Boehringer, Cardio3Biosciences, GSK, Merck/MSD, Novartis, Servier, Sphingotec, Stealth, Trevena, Vifor. Juan-I Pérez-Calvo has received speaker fees and travel/accommodation expenses covered by Novartis. Jorge Rubio-Gracia has received travel/accommodation expenses covered by Novartis. All other authors reported that they have no conflict of interest to declare.

\section{References}

[1] Yancy CW, Fonarow GC, Committee ASA. Quality of care and outcomes in acute decompensated heart failure: The ADHERE registry. Curr Heart Fail Rep. 2004; 1: 121128.

[2] Gheorghiade M, Filippatos G, De Luca L, Burnett J. Congestion in acute heart failure syndromes: an essential target of evaluation and treatment. Am J Med. 2006; 119: S3S10. 
[3] Verbrugge FH, Grieten L, Mullens W. Management of the cardiorenal syndrome in decompensated heart failure. Cardiorenal Med. 2014; 4: 176-88.

[4] Lucas C, Johnson W, Hamilton MA et al. Freedom from congestion predicts good survival despite previous class IV symptoms of heart failure. Am Heart J. 2000; 140: 840-7.

[5] Ponikowski P, Voors AA, Anker SD et al. 2016 ESC Guidelines for the diagnosis and treatment of acute and chronic heart failure. Eur J Heart Fail. 2016; 18: 891-975

[6] Lala A, McNulty SE, Mentz RJ et al. Relief and recurrence of congestion during and after hospitalization for acute heart failure: Insights From Diuretic Optimization Strategy Evaluation in Acute Decompensated Heart Failure (DOSE-AHF) and Cardiorenal Rescue Study in Acute Decompensated Heart Failure (CARESS-HF). Circ Heart Fail. 2015; 8: 741-8.

[7] Ambrosy AP, Pang PS, Khan $S$ et al. Clinical course and predictive value of congestion during hospitalization in patients admitted for worsening signs and symptoms of heart failure with reduced ejection fraction: findings from the EVEREST trial. Eur Heart J. 2013; 34: 835-43.

[8] Voors AA, Ter Maaten JM. Tackling Early Heart Failure Deaths and Readmissions by Estimating Congestion. JACC Heart Fail. 2015; 3: 894-5.

[9] Metra M, Nodari S, Parrinello G et al. Serial changes and independent prognostic value of NT-proBNP and cardia Troponin-T. Eur J Heart Fail. 2007; 9:776-86 
[10] Platz E, Merz AA, Jhund PS et al. Dynamic changes and prognostic value of pulmonary congestion by lung ultrasound in acute and chronic heart failure: a systematic review. Eur J Heart Fail. 2017; 30. [Epub ahead of print].

[11] Massie BM, O'Connor CM, Metra M et al. PROTECT Investigators and Committees. Rolofylline, an adenosine A1-receptor antagonist, in acute heart failure. N Engl J Med. 2010; 363: 1419-28.

[12] ter Maaten JM, Valente MA, Damman K et al. Diuretic response in acute heart failure-pathophysiology, evaluation, and therapy. Nat Rev Cardiol. 2015;12:184-92.

[13] Voors AA, Davison BA, Teerlink JR et al. Diuretic response in patients with acute decompensated heart failure: characteristics and clinical outcome--an analysis from RELAX-AHF. Eur J Heart Fail. 2014; 16: 1230-40.

[14] Cotter G, Dittrich HC, Weatherley BD et al. Protect Steering Committee, Investigators, and Coordinators. The PROTECT pilot study: a randomized, placebocontrolled, dose-finding study of the adenosine A1 receptor antagonist rolofylline in patients with acute heart failure and renal impairment. J Card Fail. 2008; 14: 631-40.

[15] Cleland JG, Chiswell K, Teerlink JR et al. Predictors of postdischarge outcomes from information acquired shortly after admission for acute heart failure: a report from the Placebo-Controlled Randomized Study of the Selective A1 Adenosine Receptor Antagonist Rolofylline for Patients Hospitalized With Acute Decompensated Heart Failure and Volume Overload to Assess Treatment Effect on Congestion and Renal Function (PROTECT) Study. Circ Heart Fail. 2014; 7: 76-87. 
[16] Drazner MH, Rame JE, Stevenson LW, Dries DL. Prognostic importance of elevated jugular venous pressure and a third heart sound in patients with heart failure. $\mathrm{N}$ Engl J Med. 2001; 345: 574-81.

[17] Mullens W, Abrahams Z, Francis GS et al. Importance of venous congestion for worsening of renal function in advanced decompensated heart failure. J Am Coll Cardiol. 2009; 53: 589-96.

[18] ter Maaten JM, Dunning AM, Valente MA et al. Diuretic response in acute heart failure-an analysis from ASCEND-HF. Am Heart J. 2015; 170: 313-21.

[19] Damman K, Kjekshus J, Wikstrand J et al. Loop diuretics, renal function and clinical outcome in patients with heart failure and reduced ejection fraction. Eur J Heart Fail. 2016 Mar; 18: 328-36

[20] Metra M, Davison B, Bettari L et al. Is worsening renal function an ominous prognostic sign in patients with acute heart failure? The role of congestion and its interaction with renal function. Circ Heart Fail. 2012; 5: 54-62.

[21] Mullens W, Abrahams Z, Francis GS et al. Importance of venous congestion for worsening of renal function in advanced decompensated heart failure. J Am Coll Cardiol. 2009; 53: 589-96.

[22] Damman K, van Deursen VM, Navis G et al. Increased central venous pressure is associated with impaired renal function and mortality in a broad spectrum of patients with cardiovascular disease. J Am Coll Cardiol. 2009; 53: 582-8. 
[23] van Veldhuisen DJ, Ruilope LM, Maisel AS, Damman K. Biomarkers of renal injury and function: diagnostic, prognostic and therapeutic implications in heart failure. Eur Heart J. 2016; 37: 2577-85.

[24] Gheorghiade M, Filippatos G, De Luca L, Burnett J. Congestion in acute heart failure syndromes: an essential target of evaluation and treatment. Am J Med. 2006; 119: S3-S10.

[25] Demissei BG, Valente MA, Cleland JG et al. Optimizing clinical use of biomarkers in high-risk acute heart failure patients. Eur J Heart Fail. 2016; 18: 269-80.

[26] Colombo PC, Onat D, Harxhi A et al. Peripheral venous congestion causes inflammation, neurohormonal, and endothelial cell activation. Eur Heart J. 2014; 35: 448-54.

[27] Tavazzi L, Maggioni AP, Borer JS. Should we revise our approach to 'optimal medical therapy'? The case of chronic heart failure. Eur Heart J. 2013; 34: 2792-4.

[28] Valente $M$, Voors AA, Damman $K$, et al. Diuretic response in acute heart failure: clinical characteristics and prognostic significance. Eur Heart J. 2014; 35: 1284-93.

[29] Duarte K, Monnez JM, Albuisson E, et al. Prognostic value of estimated plasma volume in heart failure. JACC Heart Fail. 2015; 3: 886-93. 


\section{TABLES}

Table 1: Baseline characteristics by CCS at Day 7

\begin{tabular}{|c|c|c|c|c|}
\hline & $\begin{array}{l}\text { NOT CONGESTION } \\
(\mathrm{CCS}=0)\end{array}$ & $\begin{array}{l}\text { MILD CONGESTION } \\
\quad(C C S=1 \text { or } 2)\end{array}$ & $\begin{array}{l}\text { SIGNIFICANT CONGESTION } \\
\text { (CCS } \geq 3 \text { ) }\end{array}$ & P-Value \\
\hline Total [n(\%)] & $370(23.5)$ & $751(47.8)$ & $451(28.7)$ & \\
\hline Age (years) & $69.6 \pm 11.9$ & $70.33 \pm 11.1$ & $70.8 \pm 11.2$ & 0.362 \\
\hline Male[n(\%)] & $239(64.6)$ & $503(67.0)$ & $300(66.5)$ & 0.724 \\
\hline BMI (Kgs/m²) & $27.0(23.7-30.4)$ & $27.1(24.2-31.1)$ & $29.5(26.1-34.5)$ & $<0.001$ \\
\hline Systolic BP at screening ( $\mathrm{mmHg}$ ) & $126.5(110.0-140.0)$ & $124.0(110.0-140.0)$ & $120.0(109.0-138.0)$ & 0.011 \\
\hline $\begin{array}{l}\text { Diastolic BP at screening } \\
\text { (mmHg) }\end{array}$ & $75.0(70.0-80.0)$ & $75.0(68.0-80.0)$ & $71.0(63.0-80.0)$ & 0.017 \\
\hline Heart rate at screening (b.p.m) & $80.0(70.0-90.0)$ & $78.0(69.0-90.0)$ & $78.0(69.0-89.0)$ & 0.121 \\
\hline NYHA [n(\%)] & & & & 0.002 \\
\hline$\bullet \quad I / I I$ & $82(23.6)$ & $104(14.6)$ & $70(16.1)$ & \\
\hline - III & $155(44.5)$ & $369(51.8)$ & $239(54.8)$ & \\
\hline - IV & $111(31.9)$ & $240(33.7)$ & $127(29.1)$ & \\
\hline$J V D>10 \mathrm{~cm}[\mathrm{n}(\%)]$ & $111(30.2)$ & $288(38.9)$ & $221(50.6)$ & $<0.001$ \\
\hline Rales more than $2 / 3$ [n(\%)] & $34(9.2)$ & $62(8.3)$ & $60(13.3)$ & 0.001 \\
\hline Edema +3 [n(\%)] & $58(15.7)$ & $161(21.4)$ & $206(45.7)$ & $<0.001$ \\
\hline Orthopnea >30 degrees [n(\%)] & $122(33.2)$ & $291(39.4)$ & $251(56.5)$ & $<0.001$ \\
\hline $\begin{array}{l}\text { Baseline Composite congestion } \\
\text { score }\end{array}$ & $5(3-6)$ & $5(4-6)$ & $6(5-7)$ & $<0.001$ \\
\hline Rolofylline treatment & $248(67.0)$ & 505 (47.9) & $302(67.0)$ & 0.994 \\
\hline \multicolumn{5}{|l|}{ HF treatment [n(\%)] } \\
\hline - ACE or ARB & $289(78.1)$ & $581(77.4)$ & 319 (70.7) & 0.016 \\
\hline - B-blockers & $274(74.1)$ & $580(77.2)$ & $363(80.5)$ & 0.089 \\
\hline - $\quad$ MRA & $157(42.4)$ & $331(44.1)$ & $190(42.1)$ & 0.767 \\
\hline - Digoxin & $115(31.1)$ & $205(27.3)$ & $138(30.6)$ & 0.305 \\
\hline \multicolumn{5}{|l|}{ History of: [n(\%)] } \\
\hline - Congestive HF & $348(94.1)$ & 713 (94.9) & 437 (96.9) & 0.132 \\
\hline - Hypertension & $293(79.2)$ & $591(78.7)$ & $373(82.7)$ & 0,222 \\
\hline - Hyperlipidemia & $162(43.8)$ & $391(52.1)$ & $267(59.3)$ & $<0.001$ \\
\hline $\begin{array}{l}\text { - } \begin{array}{l}\text { Peripheral Vascular } \\
\text { disease }\end{array} \\
\end{array}$ & $39(10.6)$ & $72(9.6)$ & $52(11.6)$ & 0.549 \\
\hline $\begin{array}{l}\text { - Asthma/Bronchitis/COP } \\
\text { D }\end{array}$ & $72(19.5)$ & $142(18.9)$ & $100(22.3)$ & 0.334 \\
\hline - Diabetes Mellitus & $144(38.9)$ & $330(44.0)$ & $245(54.3)$ & $<0.001$ \\
\hline - $\quad$ Angina & $93(25.1)$ & $173(23,0)$ & $79(17.6)$ & 0,020 \\
\hline - ICD & $40(10.8)$ & $104(13.8)$ & $110(24.4)$ & $<0.001$ \\
\hline - IHD & $234(63.2)$ & $547(73.0)$ & $307(68.2)$ & 0.003 \\
\hline - $\mathrm{MI}$ & $169(45.7)$ & $391(52.3)$ & $213(47.3)$ & 0.071 \\
\hline - $\quad$ CABG & $46(12.5)$ & $174(23.4)$ & $123(27.5)$ & $<0.001$ \\
\hline - $\mathrm{PCl}$ & $81(22.1)$ & $195(26.2)$ & $129(28.8)$ & 0.096 \\
\hline - Atrial Fibrillation/Flutter & $185(50.3)$ & $388(52.1)$ & $272(60.9)$ & 0.003 \\
\hline - $\quad$ Pacemaker & $32(8.6)$ & $88(11.7)$ & $63(14.1)$ & 0.056 \\
\hline - Biventricular Pacing & $21(5.7)$ & $70(9.3)$ & 75 (16.6) & $<0.001$ \\
\hline
\end{tabular}

Values are expressed as mean \pm SD or Median $\left(25^{\text {th }}, 75^{\text {th }}\right)$. BMI, Body Mass Index; NYHA, New York Heart Assosiation; JVD, jugular venous distension; HF, Heart Failure; ACE, Angiotensin-converting-enzyme inhibitors; ARB, Angiotensin II receptor blocker; MRA, mineralocorticoid receptor antagonist; COPD, Chronic obstructive pulmonary disease; ICD, implantable cardioverter-defibrillator; ICP, Internal Cardiac Desfibrillator;; IHD, Ischemic Heart Disease; MI, myocardial Infarction; $\mathrm{CABG}$, Coronary Artery Bypass Graft; $\mathrm{PCl}$, Percutaneous Coronary Intervention. 


\begin{tabular}{|c|c|c|c|c|}
\hline & $\begin{array}{l}\text { NOT CONGESTION } \\
(C C S=0)\end{array}$ & $\begin{array}{l}\text { MILD CONGESTION } \\
\text { (CCS=1 or } 2)\end{array}$ & $\begin{array}{l}\text { SIGNIFICANT } \\
\text { CONGESTION } \\
\text { (CCS } \geq 3 \text { ) }\end{array}$ & P-Value \\
\hline LVEF (\%) & $32.0(23.5-41.5)$ & $30.0(22.5-40.0)$ & $27.7(20.0-40.0)$ & 0.044 \\
\hline BUN (mg/dL) & $26.0(21.0-36.0)$ & $28.0(21.0-39.0)$ & $35.0(24.0-48.5)$ & $<0.001$ \\
\hline Creatinine $(\mathrm{mg} / \mathrm{dL})$ & $1.3(1.10-1.60)$ & $1.4(1.1-1.7)$ & $1.5(1.2-1.9)$ & $<0.001$ \\
\hline $\begin{array}{l}\text { eGFR } \\
\left(\mathrm{mL} / \mathrm{min} / 1.73 \mathrm{~m}^{2}\right)\end{array}$ & $49.5(38.1-66.6)$ & $48.4(36.0-64.0)$ & $42.6(32.4-58.4)$ & $<0.001$ \\
\hline Hematocrit (\%) & $41.0(37.4-44.8)$ & $40.0(35.9-44.2)$ & 38.5 (34.9-42.9) & $<0.001$ \\
\hline Hemoglobin (g/dL) & 13.1 (11.8-14.4) & $12.6(11.3-13.9)$ & $12.0(10.9-13.5)$ & $<0.001$ \\
\hline Glucose & $121.0(98.5-158.0)$ & $127.0(103.0-164.0)$ & $128.0(103.0-168.0)$ & 0.048 \\
\hline Albumin & $3.9(3.7-4.2)$ & $3.9(3.6-4.1)$ & $3.7(3.5-4.1)$ & $<0.001$ \\
\hline Sodium (mmol/L) & $141.0(138.0-143.0)$ & $140.0(137.0-142.0)$ & $139.0(136.0-142.0)$ & $<0.001$ \\
\hline $\begin{array}{l}\begin{array}{l}\text { Potassium } \\
\text { (mmol/L) }\end{array} \\
\end{array}$ & $4.2(3.9-4.6)$ & $4.2(3.8-4.6)$ & $4.2(3.8-4.6)$ & 0.514 \\
\hline Chloride (mmol/L) & $102.0(100.0-105.0)$ & $101.0(98.0-104.0)$ & $100.0(97.0-100.4)$ & $<0.001$ \\
\hline $\begin{array}{l}\text { Bicarbonate } \\
\text { (mmol/L) }\end{array}$ & $24.0(21.0-26.0)$ & $24.0(21.0-26.0)$ & $24.0(21.0-27.0)$ & 0.540 \\
\hline Cholesterol & $152.0(125.5-182.0)$ & $143.0(117.0-175.2)$ & $127.0(104.0-160.0)$ & $<0.001$ \\
\hline Triglycerides & $90.0(66.0-137.0)$ & $91.0(66.0-125.0)$ & $82.0(64.0-112.0)$ & 0.013 \\
\hline BNP (ng/L) & $479.0(264.6-799.6)$ & $425.46(258.7-771.3)$ & $502.4(263.4-915.6)$ & 0.046 \\
\hline $\begin{array}{l}\text { Total dose of IV } \\
\text { loop diuretic until } \\
\text { day } 7 \text { or discharge } \\
\text { (mg of furosemide) }\end{array}$ & $209(100-365)$ & $240(120-491)$ & $400(200-920)$ & $<0.001$ \\
\hline $\begin{array}{l}\text { Study day of } \\
\text { discharge }(n)\end{array}$ & $8(7-14)$ & $8(5-14)$ & $9(5-16)$ & 0.108 \\
\hline
\end{tabular}

All values are expressed as Median $\left(25^{\text {th }}, 75\right.$ th). LVEF, Left Ventricular Ejection Fraction; BUN, Blood urea nitrogen; eGFR, Estimated Glomerular Filtration rate calculated by the simplified modification of diet in renal disease equation,; BNP, Brain Natriuretic Peptide; 
Table 2: Factors associated with the presence of significant residual congestion by day 7; a multivariable logistic regression model.

\begin{tabular}{|c|c|c|c|}
\hline Variable & Z-Score & OR $[95 \% \mathrm{Cl}]$ & P-value \\
\hline $\begin{array}{l}\text { Composite Congestion } \\
\text { Score }\end{array}$ & 69.7 & $1.70[1.50-1.92]$ & $<0.001$ \\
\hline BUN & 22.3 & $1.48[1.25-1.74]$ & $<0.001$ \\
\hline Diuretic response & 17.9 & $1.65[1.31-2.09]$ & $<0.001$ \\
\hline BMI & 17.3 & $1.45[1.22-1.73]$ & $<0.001$ \\
\hline Total Cholesterol & 9.7 & $0.75[0.63-0.90]$ & 0.002 \\
\hline $\operatorname{Ln}(A L T)$ & 7.1 & $0.79[0.67-0.94]$ & 0.008 \\
\hline History of Pacemaker & 6.8 & $1.89[1.17-3.04]$ & 0.009 \\
\hline BNP & 6.5 & $1.23[1.05-1.44]$ & 0.010 \\
\hline
\end{tabular}

Factors have been ordered by Z-Score

AUC for this multivariable model is 0.80

CCS, Composite Congestion Score; BUN, Blood Urea Nitrogen; BMI, Body Mass Index; BNP, Brain Natriuretic Peptide. For continuous variables ORs should be interpreted per SD. 